\title{
LA QUERATOMETRÍA COMO VARIABLE PREDICTORA DE LA AMETROPÍA: UN ESTUDIO DIAGNÓSTICO EN LA POBLACIÓN ESCOLARIZADA DE 5 A 19 AÑOS DE LA CIUDAD DE PEREIRA
}

Héctor Aníbal Sánchez Montaña', Paulo Cesar Zapata Giraldo², Yuli Carolina

Delgado Solarte ${ }^{3}$, Maryury Potes Flórez ${ }^{4}$

\section{Resumen}

Objetivo: determinar la relación entre la curvatura corneal y los defectos de refracción en la población estudiantil de 5 a 19 años de la ciudad de Pereira en el año 2014. Materiales y métodos: el tipo de estudio esobservacional correlacional descriptivo. Se indica el modelo de regresión lineal simple entre la queratometría y la retinoscopía. Se evaluó el error refractivo en 915 estudiantes de Pereira medianteretinoscopía estática y queratometría. Resultados: el valor cilíndrico obtenido para el ojo derecho (OD) oscila entre $-0,12$ y $-5,50 \mathrm{dpt}$, mientras que para el ojo izquierdo (OI), el valor oscila entre $-0,12$ y $-6,12$ dpt. El 86,6\% del meridiano eje (ME) del OD está entre 38,00 y 43,88 dpt, en tanto que el $87,2 \%$ del ME del OI se encuentra entre 32,00 y 43,88 dpt. La ametropía positiva para el OD está entre 0,25 y $8,00 \mathrm{dpt}$; para el OI en 0,25 y 7,50dpt. La ametropía negativa está en 6,00 para el OD y 6,50 dpt para el OI como valores máximos. En relación a la curvatura corneal y a las ametropías, el OD muestra una correlación de Pearson

${ }^{1}$ Optómetra y Magíster en Comunicación Educativa, Universidad Tecnológica de Pereira. Docente e integrante del grupo de investigación Salud Visual, Fundación Universitaria del Área Andina, seccional Pereira.

${ }^{2}$ Optómetra y Magíster en Ciencias de la Visión, Universidad de La Salle. Docente y director del grupo de investigación Salud Visual, Fundación Universitaria del Área Andina, seccional Pereira.

${ }^{3}$ Estudiante de optometría, décimo semestre. Fundación Universitaria del Área Andina, seccional Pereira.

${ }^{4}$ Estudiante de optometría, décimo semestre. Fundación Universitaria del Área Andina, seccional Pereira. 
negativa, de $-0,21(\mathrm{p}=0.0001)$. En el OI la correlación de Pearson también es negativa, de $-0,20(\mathrm{p}=0.0001)$. Por lo tanto, la relación encontrada entre estas dos variables es tan solo del $5,1 \%$ y un $4,7 \%$ para OD y OI, respectivamente. Conclusiones: la relación entre la curvatura corneal y los errores de refracción según este modelo solo explica un porcentaje insignificante que no tiene importancia clínica, es del 4,9\% en promedio del valor obtenido en el error refractivo en ambos ojos. Por ello, no es posible hacer una predicción de los errores refractivos con base en el radio de la curvatura corneal. 


\section{THE KERATOMETRY AS A PREDICTOR VARIABLE OF AMETROPY: A DIAGNOSTIC STUDY IN SCHOOLED POPULATION BETWEEN THE AGES OF 5 TO 19 IN THE CITY OF PEREIRA}

Héctor Aníbal Sánchez Montaña, Paulo Cesar Zapata Giraldo², Yuli Carolina Delgado Solarte ${ }^{3}$, Maryury Potes Flórez ${ }^{4}$

\section{Abstract}

Objective: The purpose of this research was to determine the relationship between corneal curvature and refractive errors in schooled population between the ages of 5 to 19 in the city of Pereira in 2014. Materials and Methods: This is a descriptive correlational observational study, in which the simple linear regression model between keratometry and retinoscopy is indicated. Through static retinoscopy and keratometry, refractive error was evaluated in 915 students of Pereira. Results: The cylindrical value obtained for the RE (right eye) oscillates between -0.12 and $-5.50 \mathrm{dpt}$ and for the LI (left eye) -0.12 and -6.12 dpt, where $86.6 \%$ of the Axis (ME in Spanish) RE is between 38.00 and 43.88dpt, while $87.2 \%$ of Axis LE is between 32.00 and $43.88 \mathrm{dpt}$. The positive ametropia for the RE is between 0.25 and $8.00 \mathrm{dpt}$, for the LE 0.25 and 7.50dpt, the negative in RE 6.00 and OI 6.50dpt as maximum values. In relation to corneal curvature and ametropias, the RE shows a negative Pearson correlation of $-0.21(p=0.0001)$. In the LE the Pearson correlation is also negative, of $-0.20(p=0.0001)$. Therefore, the relationship found between these two variables is only $5.1 \%$ and $4.7 \%$ for RE and LE respectively. Conclusions: The relationship between corneal curvature and refractive errors according to this model only explains an insignificant percentage that does not have clinical importance, it is a $4.9 \%$ on average of the value obtained in the refractive error in both eyes, therefore, it does not allow a prediction of refractive errors based on the radius of corneal curvature. 


\section{CERATOMETRIA COMO UMA VARIÁVEL PREDITIVA DE AMETROPIA: UM ESTUDO DIAGNÓSTICO NA POPULAÇÃO ESCOLAR DE 5 A 19 ANOS DE CIDADE DE PEREIRA}

Héctor Aníbal Sánchez Montaña, Paulo Cesar Zapata Giraldo, Yuli Carolina Delgado Solarte, Maryury Potes Flórez

\section{Resumo}

Objetivo. $\mathrm{O}$ objetivo desta pesquisa foi determinar a relação entre a curvatura da córnea e erros de refração na população estudantil de 5 a 19 anos de idade na cidade de Pereira em 2014. Materiais e Métodos. O tipo de estudo é descritivo correlacional observacional, no qual o modelo de regressão linear simples entre ceratometria e retinoscopia é indicado. Por meio de retinoscopia estática e ceratometria, o erro refrativo foi avaliado em 915 estudantes de Pereira. Resultados. O valor cilíndrico obtido para o OD (olho direito) é entre -0,12 e -5,50dpt e para o OI (olho esquerdo) e - 0,12 dpt e $-6,12$, onde $86,6 \%$ do Meridiano Eje (ME) OD está entre 38,00 e 43,88dpt, enquanto que 87,2\% do ME OI está entre 32,00 e 43,88dpt. A ametropia positiva para o OD está entre 0,25 e 8,00dpt, para o OI 0,25 e 7,50dpt, a ametropia negativa em OD 6,00 e OI 6,50dpt como valores máximos. Em relação à curvatura corneana e ametropias, o OD mostra uma correlação negativa de Pearson de $-0,21(\mathrm{p}=0,0001)$. No OI, a correlação de Pearson também é negativa, de $-0,20(\mathrm{p}=0,0001)$. Portanto, a relação encontrada entre essas duas variáveis é de apenas 5,1\% e 4,7\% para OD e OI, respectivamente. Conclusões. A relação entre a curvatura corneana e os erros refrativos de acordo com este modelo explica apenas uma percentagem insignificante de nenhuma importância clínica, é de 4,9\%, em média, do valor obtido no erro refrativo em ambos os olhos, de modo que, não permite fazer uma previsão de erros refrativos com base no raio da curvatura corneana. 


\section{Introducción}

Según la Organización Mundial de la Salud (OMS), en su plan de acción mundial 2014 a 2019 sobre la salud ocular universal, existen millones de personas ciegas y con déficit visual en todo el mundo, principalmente en los países en vía de desarrollo. Por lo tanto, se requiere de programas de salud y desarrollo, investigaciones y la potenciación de los recursos humanos para reducir la discapacidad visual evitable. Un registro realizado por el Departamento Administrativo Nacional de Estadística (DANE) revela, a marzo del 2009, 231072 personas con discapacidad visual(1).

Una de las acciones fundamentales del trabajo clínico en optometría que le compete al profesional de la salud visual es realizar diagnósticos tempranos del estado refractivo del paciente mediante diferentes exámenes. Para efectos de la presente investigación, se tendrán en cuenta variables indispensables como la queratometría y la retinoscopía para determinar el error refractivo y la curvatura corneal(2).

Ametropía es una palabra que procede del griego ametro, "sin medida» y ops, «ojo»(3). Entre estas se encuentra la hipermetropía y miopía, cuyos rayos luminosos provenientes del infinito son enfocados por detrás o delante de la retina. Por tal motivo la imagen se ve desenfocada, así que se genera un desequilibrio entre la potencia del ojo y la longitud axial(4)(5). El astigmatismo es una ametropía y ocurre cuando el sistema óptico no puede formar imágenes en un foco definido, debido a que los dos meridianos principales generan dos lí- neas focales en distintos puntos de la retina, las cuales generan un recorrido denominado Conoide de Sturm, es decir, se forman círculos de difusión retinal en el sistema óptico astigmático, los cuales generan una imagen desenfocada. Hacia el centro se forma el círculo de menor confusión, en el sitio donde la agudeza visual puede ser mejor(6). El radio de curvatura corneal anterior determina dos tercios del poder de la refracción óptica del ojo y es, por tanto, un parámetro importante del sistema óptico ocular; cualquier disparidad entre los meridianos de la córnea producen astigmatismo corneal(7).

En las ametropías se destaca la longitud axial como factor indispensable para su determinación: si es mayor al promedio, se tiende a ser miope; si es inferior, hipermétrope, según el ojo esquemático, en el que la longitud axial es de aproximadamente $23 \mathrm{~mm}$. No obstante, la longitud axial no es el único factor que influye en los defectos refractivos, también lo hacen el poder y la curvatura de la córnea, que es lo que tiene por objeto la presente investigación(8).

Las variables biométricas principales que contribuyen potencialmente a un estado de refracción son los poderes de la córnea, el cristalino, la cámara anterior y la cámara vítrea. Los análisis de correlación han demostrado que los componentes oculares principales que influyen en el error de refracción son interdependientes y que, durante el desarrollo temprano, crecen de forma coordinada, razón por la cual el globo ocular puede tender a la emetropía. De este modo, por ejemplo, el aumento de la longitud axial 
en el desarrollo temprano contrarresta el poder de la córnea, puesto que disminuye concomitantemente(9).

Por lo general, el inicio y la progresión de la miopía están fuertemente correlacionados con el aumento de longitud axial y, en concreto, con la profundidad de la cámara vítrea. Sin embargo, hay poca -o ninguna- evidencia de que aumente en potencia corneal. Por ello, es necesario estudiar la relación entre la potencia de la córnea, que es determinada por la curvatura y los diferentes defectos de refracción(8).

En Colombia, las investigaciones respecto a la curvatura corneal y defectos refractivos se desconocen, a pesar de que es un factor indispensable para la determinación de los defectos refractivos y su corrección. El presente estudio se plantea ante dicha necesidad; se encontró tan solo un estudio en China de los defectos refractivos y la curvatura corneal(10). Por lo tanto, al realizar el diagnostico refractivo del paciente, se debe tener en cuenta la integralidad del sistema óptico y tener como referente la curvatura corneal para determinar el astigmatismo, mas no el estado refractivo esférico.

\section{Materiales y métodos}

El tipo de estudio realizado es de corte transversal y permitió determinar la relación entre la curvatura corneal y las ametropías en 915 estudiantes entre los 5 y 19 años de los colegios públicos de Pereira.

Se estimó un tamaño de muestra según lo recomendado por McAlinden et al., basado en la precisión deseada del intervalo de confianza para la estimación de los límites, de acuerdo con Bland y Altman, así: desviación estándar de las diferencias de 0,72, confiabilidad de 95\% y una precisión de 0,25 dpt" (11).

Los criterios para la selección de la población de estudio que se tuvieron en cuenta en la investigación "Concordancia entre el astigmatismo según las leyes de Javal y la refracción subjetiva" fueron de inclusión y exclusión. Se incluyeron estudiantes hombres y mujeres de los colegios públicos ${ }^{1}$ de Pereira con defectos refractivos que asistieron a control o consulta de primera vez entre los 5 y 19 años.

Se excluyeron estudiantes con patologías $\mathrm{u}$ opacidades en córnea que impidan hacer la queratometría, enfermedades sistémicas no controladas, antecedentes de cirugías refractivas, con disminución de capacidades cognitivas, ojo único, astigmatismos irregulares y mujeres en embarazo, ya que su estado refractivo es variable durante esta etapa(12).

Los valores se extrajeron de una base de datos resultado de una investigación previa que reunía los resultados de la queratometría con queratómetro de Helmholtz, la cual se realizó de acuerdo con un protocolo previamente establecido y la refracción objetiva. Estos datos fueron los que se usaron para hacer la relación y obtener los resultados de la investigación. Por otro lado,

1 Los colegios fueron: Kennedy, Luis Alfredo, Carlota Sánchez, Byron Gaviria, San Vicente Hogar, Gimnasio Risaralda, Francisco de Paula Santander, Jorge Eliecer Gaitán, Pablo Emilio Cardona y Augusto Zuluaga. 
el análisis de las variables cuantitativas se realizó a través del paquete de software estadístico Stata 13.0 y la aplicación de Excel. Para este caso, el modelo de regresión lineal mostró que entre la variable curvatura corneal y ametropías se obtuvo un coeficiente de $-0,16$, donde se evidenció un cambio en promedio de 0,16 dioptrías en el defecto refractivo por cada dioptría de cambio en la curvatura corneal.

\section{Resultados}

Al caracterizar sociodemográficamente a la población, se encontró que el promedio de edad era de 11,5 años. El 43,1\% son de género masculino y el $56,9 \%$ femenino, es decir, 394 son hombres y 521 mujeres.

Tabla 1. Curvatura corneal.

\section{Curvatura corneal}

La población objeto de estudio mostró, para el ojo de derecho, un valor queratométrico mínimo de 38,00 dioptrías y uno máximo de 47,25 dioptrías; para el ojo izquierdo fue uno mínimo de 32,25 dioptrías y uno máximo de 47,75 dioptrías. El defecto astigmático oscila entre -0,12 y -5,50 dioptrías de cilindro para el ojo derecho, mientras que, para el ojo izquierdo, el valor cilíndrico más alto encontrado es de -6,12. El registro de la queratometría se realizó en pasos de 0,12 dioptrías para mayor exactitud en la toma de la prueba.

A continuación, se presentan en las Tablas 1 y 2 los porcentajes obtenidos en queratometría para cada ojo, de acuerdo con el meridiano más plano:

\begin{tabular}{c|c|c|c}
\hline $\begin{array}{c}\text { Meridiano eje ojo } \\
\text { derecho (dpt) }\end{array}$ & Porcentaje (\%) & $\begin{array}{c}\text { Meridiano eje ojo } \\
\text { izquierdo (dpt) }\end{array}$ & Porcentaje (\%) \\
\hline $32,00-33,88$ & 0 & $32,00-33,88$ & 0,1 \\
$34,00-35,88$ & 0 & $34,00-35,88$ & 0,1 \\
$36,00-37,88$ & 0 & $36,00-37,88$ & 0 \\
$38,00-39,88$ & 6,1 & $38,00-39,88$ & 6,9 \\
$40,00-41,88$ & 35,4 & $40,00-41,88$ & 35,8 \\
$42,00-43,88$ & 45,1 & $42,00-43,88$ & 44,3 \\
44.00 & 3,8 & 44.00 & 3,2 \\
$44,12-45,00$ & 7,1 & $44,12-45,00$ & 6,8 \\
$45,12-46,00$ & 2,0 & $45,12-46,00$ & 2,0 \\
$46,12-47,00$ & 0,4 & $46,12-47,00$ & 0,5 \\
$47,12-48,00$ & 0,1 & $47,12-48,00$ & 0,3 \\
Total & 100 & Total & 100 \\
\hline
\end{tabular}

Fuente: Elaboración propia. 
En la tabla se pude observar que, para el ojo derecho, el 86,6\% de la población tiene cornea plana y para el ojo izquierdo es un 87,2\%. El 9,6\% corresponde a córneas curvas para cada ojo, mientras que el porcentaje de córneas normales para el ojo derecho es de 3,8\% y para el ojo izquierdo es de $3,2 \%$.

\section{Determinación de ametropías}

Para la determinación del defecto refractivo se toma el valor esférico obtenido en la retinoscopía estática, para la que se obtuvieron valores, para el ojo derecho, de hipermetropía desde 0,25 hasta 8,00 dioptrías como máximo, y de miopía desde 0,25 y hasta 6,00 dioptrías. En contraste, el ojo izquierdo mostró un valor máximo de hipermetropía de 0,50 dioptrías menos, es decir, 7,50 dioptrías, y para miopía de 6,50 dioptrías, es decir, 0,50 dioptrías más que para el ojo derecho. En la siguiente tabla se muestra la clasificación de las ametropías y los porcentajes de los defectos refractivos obtenidos(13):

Tabla 2. Defecto refractivo

\begin{tabular}{l|c|c|c}
\hline \multicolumn{1}{c|}{ Clasificación } & Valor esférico en dpt & $\begin{array}{c}\text { Porcentaje ojo } \\
\text { derecho }\end{array}$ & $\begin{array}{c}\text { Porcentaje ojo } \\
\text { izquierdo }\end{array}$ \\
\hline Miopía alta & $(-7,00)-(-9,00)$ & 0 & 0 \\
Miopía media & $(-6,00)-(-4,00)$ & 0,9 & 0,5 \\
Miopía baja & $(-0,25)-(-3,00)$ & 14,4 & 13,3 \\
Emétrope & $(0,0)$ & 13,6 & 13,5 \\
Hipermetropía baja & $(+0,25)-(+2,00)$ & 66,4 & 67,2 \\
Hipermetropía media & $(+2,25)-(+5,00)$ & 4,1 & 4,8 \\
Hipermetropía alta & $(+5,25)-(y$ más $)$ & 0,6 & 0,7 \\
& Total & $100 \%$ & $100 \%$ \\
\hline
\end{tabular}

Fuente: Elaboración propia.

\section{Curvatura corneal vs. Ametropías}

Tabla 3.

\begin{tabular}{lcc}
\hline \multicolumn{1}{c|}{ Variable independiente } & Coeficiente & Valor $\mathbf{p}$ \\
\hline Curvatura corneal OD & -0.16 & 0.000 \\
Curvatura corneal OI & -0.15 & 0.000 \\
\hline
\end{tabular}


Con relación a la curvatura corneal y a las ametropías, respecto al ojo derecho se encontró que es inversa, puesto que la correlación de Pearson es negativa, dado que es $-0,21$ ( $\mathrm{p}=0.0001)$.A partir del modelo de regresión lineal entre las variables curvatura corneal y ametropías se obtiene un coeficiente de -0.16 , lo que arroja un cambio en promedio de 0,16 dioptrías en el defecto refractivo por cada dioptría de cambio en la curvatura corneal. Este modelo solo explica el 5,1\% del valor obtenido en el error refractivo. En la Figura 1, la línea diagonal sólida indica la línea de regresión lineal $($ Esf $=7,22-0,16[\mathrm{rc}])$.

Figura 1. Relación entre la curvatura corneal y las ametropías del ojo derecho.

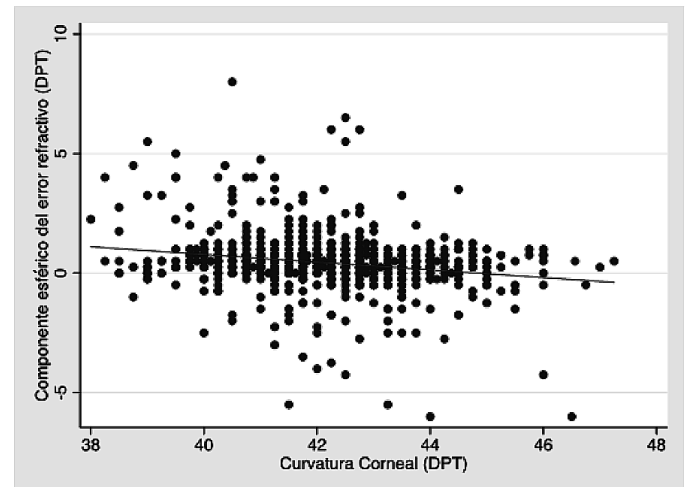

Fuente: Elaboración propia.

En relación con la curvatura corneal y a las ametropías, respecto al ojo izquierdo se encontró que es inversa, puesto que la correlación de Pearson es de $-0,20(p=0.0001)$, es decir, es negativa. Según el modelo de regresión lineal, entre las variables curvatura corneal y ametropías se obtiene un coeficiente de $-0,15$, lo que muestra un cambio en promedio alrededor de 0,15 dioptrías en el defecto refractivo por cada dioptría de cambio en la curvatura corneal. Sin embargo, este modelo solo explica el 4,7\% del valor obtenido en el error refractivo. En la Figura 2, la línea diagonal sólida indica la línea de regresión lineal $($ Esf $=6,87$ $-0,15[\mathrm{rc}])$

Figura 2. Relación entre la curvatura corneal y las ametropías del ojo izquierdo.

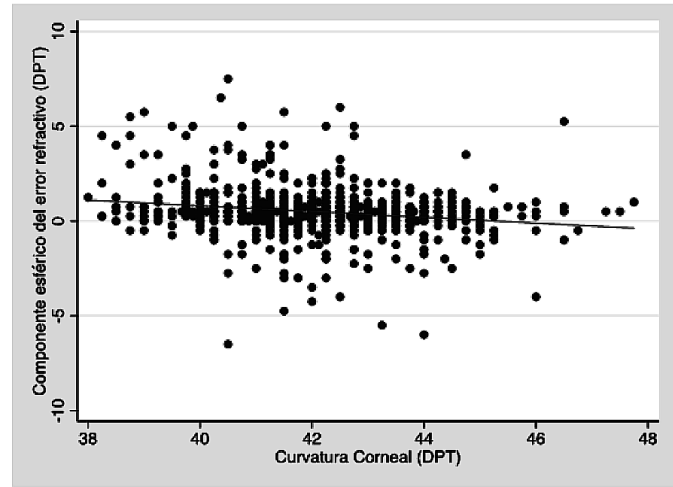




\section{Discusión}

De acuerdo con el modelo de regresión lineal, los resultados de la presente investigación indicaron que cerca de un 5,1\% y un $4,7 \%$ del error de refracción del ojo derecho e izquierdo, respectivamente, se relaciona con los valores queratométricos. Sin embargo, para la práctica clínica estos valores no son significativos, puesto que esta relación es inversa y no permite hacer una medición de ametropías teniendo como base el radio de curvatura corneal. En otras palabras, la curvatura corneal no determina el defecto de refracción, sino múltiples factores que se han mencionado a lo largo del estudio, razón por la cual una cornea plana no se puede relacionar con hipermetropía, ni una cornea curva con miopía; como se pudo observar, existen curvaturas corneales planas con defectos refractivos miópicos que contradicen la teoría del ojo esquemático y que no es aplicable a la realidad. Teniendo en cuenta que la definición del ojo esquemático pretende facilitar el estudio del ojo desde el punto de vista óptico, al atribuirle un valor dióptrico a los dos medios refringentes principales del ojo (la córnea y el cristalino), se incurre en un sesgo al decir que el poder corneal es de 44,00 dioptrías para un ojo emétrope, pues los resultados obtenidos indican que este valor no hace referencia necesariamente a emetropía(11).

Como se explica anteriormente, los hallazgos respecto a la refracción no guardan relación con estos datos queratométricos, como lo sustenta Guerrero, quien establece la correspondencia queratométrica con la esfera del estado refractivo ocular, en donde queratome- trías iguales o inferiores a 43,75 dioptrías corresponden con corneas planas, y 44,25 dioptrías a corneas curvas(14).

Por lo tanto, se puede concluir que un radio de curvatura mayor no necesariamente hace referencia a un defecto positivo y viceversa. Por ello, existen otros factores que representan el $95 \%$ de lo que se relaciona con los errores refractivos. Generalmente, las causas más importantes que dan lugar a la aparición de anomalías refractivas son alteraciones en: la longitud axial, la curvatura de las superficies refractivas y en los índices de refracción; es posible intervenir en la génesis de las ametropías esféricas de uno o varios de los factores(15).

Adicionalmente, otros estudios sugieren que la aparición de ametropías tiene que ver con la genética, tal es el caso del estudio de Feng, en el cual los estudios genéticos han identificado 18 posibles loci en 15 cromosomas diferentes que están relacionadas con la miopía. Además, se ha informado que la refracción y las características subyacentes de la longitud axial, la curvatura corneal y la profundidad de la cámara anterior son altamente heredables(16). Por su parte, los hallazgos de Quiao-girder, al inducir experimentalmente ametropías para determinar la naturaleza de estas en 210 monos Rhesus (Macaca mulatta), indicaron que el principal componente que contribuye a la aparición de ametropías es la profundidad de la cámara vítrea y que, en una menor proporción, el poder de la córnea. Con relación a la cámara anterior y al cristalino se encontró que estos no tienen relación con los errores de refracción. De este modo, este estu- 
dio demuestra que los ojos miopes se caracterizan por ejes anteroposteriores más largos, mayor profundidad de cámara vítrea y potencia corneal atribuibles al $80 \%$ y $10 \%$ de las variaciones del error refractivo, respectivamente. $\mathrm{Al}$ ser comparado con este estudio, el valor atribuible al poder corneal es inferior al $10 \%(9)$.

Wildsoet en 1998 dice que el alargamiento de la longitud axial genera miopía, al igual que Stenstrom en 1948, encontró que existe una alta relación entre la longitud axial y los errores de refracción. Van Alphen en 1961, con base en los hallazgos de Stenstrom, concluyó que un miope presenta mayor longitud axial, una cornea más potente y un cristalino más plano, pero hay poca evidencia de que el poder corneal aumente. En contraposición a esta idea, el estudio de Quiao-girder encontró que la potencia corneal se correlaciona negativamente con los componentes axiales, especialmente con la profundidad de la cámara vítrea y la longitud axial total, es decir, en teoría, cuanto mayor sea el diámetro del ojo, menor será la potencia corneal(9).

Además, resultados similares han sido reportados en los seres humanos. Koretz 1995 evaluó 185 ojos humanos y también encontró que la correlación negativa entre el poder corneal y la longitud axial eran débiles, mientras que la correlación positiva entre la longitud axial y la cámara vítrea eran más fuertes en los ojos amétropes, en comparación con los ojos emetrópicos(9).

Igualmente, Guggenheim refiere que los errores de refracción surgen debido a un desequilibrio entre el poder de enfoque del ojo y la posición de la retina; el primero se rige principalmente por la curvatura de la córnea, ya que esto proporciona dos tercios de potencia de refracción del ojo. El plano de la retina, en relación con la córnea, se determina por la longitud axial del ojo. Por ello, la hipermetropía se relaciona con longitudes axiales cortas y la miopía con longitudes axiales largas(17).

Entonces, el radio de curvatura en relación con la longitud axial indica que, a mayor longitud axial, menor radio de curvatura, lo cual se relaciona con las afirmaciones de Van Alphen, y contradicen los hallazgos de Quiao-girder. No obstante, en el caso de ametropías, se debilita la escala coordinada de los componentes de tamaño de los ojos, por lo que los cientos a miles de variantes genéticas comunes coordinan la dimensión de los componentes oculares(17).

Por su parte, Kingston estudió la relación entre la aberración esférica total de ametropía, la edad, la curvatura corneal, y la calidad de la imagen; añade que existe una relación entre la curvatura corneal y la aberración esférica, debido al aumento en el poder de la superficie anterior corneal y el grado de aberración esférica y la ametropía no se correlacionan. Muchos estudios han establecido la correlación entre la curvatura corneal y su papel en la aberración esférica, pero muy pocos han hecho la medición de la curvatura corneal, razón por la cual Kingston determinó que, a mayor poder corneal, la aberración esférica se hace más positiva(18). 
Y. Zhang afirma que la correlación de una córnea plana puede, por lo menos, de manera parcial compensar el alargamiento axial para preservar la emetropía en el crecimiento normal del ojo. En tanto que las miopías altas, con una longitud axial de más de $26,5 \mathrm{~mm}$ o un error refractivo de más de 8 dioptrías no muestran una asociación entre el radio de curvatura corneal y la longitud axial en adultos. Finalmente, un radio de curvatura menor se asoció significativamente con una mayor cantidad de astigmatismo corneal(7)

En otro estudio, Yebra-Pimentel estableció que las relaciones entre la asfericidad corneal y el error refractivo no eran significativas, pero que sí existía correlación significativa de la asfericidad con respecto al radio de curvatura, a la profundidad de la cámara vítrea y a la longitud axial. Los 109 sujetos evaluados correspondían con el patrón de córnea elipse prolata, el parámetro en seres humanos tanto en amétropes como en emétropes. Por tanto, se puede concluir que la condición refractiva difiere en su curvatura central, pero no en su configuración periférica(18).

De acuerdo con Duke-Elder, los componentes ópticos oculares más determinantes en el estado refractivo del ojo son la longitud axial, la profundidad de la cámara anterior y el espesor del cristalino, pues, como se aprecia no tiene en cuenta la curvatura corneal para la determinación de ametropías (19).
Los aportes de los diferentes estudios son significativos, dado que, por ejemplo, se encontró que el poder corneal tiene una baja relación con los defectos refractivos, según el estudio de Quiao-girder, que aporta la relación entre el radio corneal y el error refractivo. En él, se encontró una relación del 10\%, mientras que el presente estudio, que fue en humanos, fue tan solo del 4,9\% en promedio(9). A pesar de ser un estudio en un modelo animal, los autores afirman y se sustentan en otros estudios para plantear la alta concordancia entre este y el modelo humano, debido a que los mecanismos de visión son similares en las dos especies, por lo que serían aplicables y reproducibles.

\section{Conclusión}

La queratometría y la retinoscopía estática deben ser elementos fundamentales en la práctica clínica de optometría, pero se debe tener en cuenta que la medición del valor esférico va dada únicamente por la retinoscopía, en tanto que el valor cilíndrico puede basarse en la diferencia de poderes de los meridanos refringentes principales.

Adicionalmente, se debe profundizar más en el tema de la curvatura corneal, ya que la mayoría de estudios mencionados hacen referencia a la longitud axial. 


\section{Referencias}

1. Organización Mundial de la Salud (OMS). Salud ocular universal: Plan de acción mundial para 2014 - 2019. 2013.

2. Piñero $D$. The role of refraction in vision research. J Optom [Internet]. 2014 [citado 2014 Sep 23];7(3):111-2. Disponible en: http://www.pubmedcentral.nih.gov/articlerender. .cgi ?artid $=4087175 \&$ tool=pmcentrez\&rendertype $=$ abstract

3. García E, Silega Y, Aparicio A. Frecuencia de ametropías en niños. Rev Cubana Pediatr. 2010;82(3):28-37.

4. Benjamin W. Borish's Clinical Refraction [Internet]. Butterworth-Heinemann; 2006 [citado 2014 Sep 23]. 1694 p. Disponible en: http://books.google.com/books?id=Yeq8QAAACAAJ\&pgis=1

5. Bueno J, Hunter J, Campbell M. Miopía y aberraciones monocromáticas en adultos jóvenes. Gaceta óptica. 2007;413:206.

6. Gabás J. Tratamiento del astigmatismo moderado en pacientes con cataratas (tesis). Zaragoza: Universidad de Zaragoza; 2013.

7. Zhang Y, et al. Corneal Curvature Radius and Associated Factors in Chinese Children: The Shandong Children Eye Study. PLoS One [Internet]. 2015;10:e0117481. Disponible en: http://dx.plos.org/10.1371/ journal.pone. 0117481

8. Puell M. Óptica fisiológica. Madrid: Editorial Complutense; 2006. 302 p.

9. Qiao-grider Y, et al. Nature of the refractive errors in Rhesus Monkeys with experimentally induced ametropias. Vision Res. 2011;50(18):1867-81.

10. Chen M. Relationship between central corneal thockness, refractive error, corneal curvature, anterior chamber depth and axial length. J Chin Med Assoc. 2009;72(3):133-7.

11. Sánchez H, Rodríguez L. Concordancia entre el astigmatismo determinado por la ley de Javal y mediante refracción subjetiva. Fundación Universitaria del Área Andina. Pereira - Risaralda; 2012 p. 1-24.

12. Charlin C. Trastornos visuales y embarazo. Rev Med Hondur [Internet]. 1931 [citado año mes día]:52-6. Disponible en: http://www.bvs.hn/RMH/pdf/1931/pdf/A11-1931-14.pdf

13. Merchán M. Corrección de la hipermetropía simple y astigmatismo hipermetrópico en niños de 0 a 4 años. Revista ciencia y tecnología para la salud visual y ocular. 2007;105-15.

14. Guerrero J. Optometría Clínica. $2^{\mathrm{a}}$ Ed. Bogotá, D.C.:Fundación Universitaria del Área Andina, editor; 2012.

15. Guggenheim J et al. Coordinated genetic scaling of the human eye: Shared determination of axial eye length and corneal curvature. Investig Ophthalmol Vis Sci. 2013;54(3):1715-21.

16. Kingston A, Cox I. Population spherical aberration: Associations with ametropia, age, corneal curvature, and image quality. Clin Ophthalmol. 2013;7:933-8.

17. Yebra-Pimentel E, et al. Asfericidad corneal en una población de adultos jóvenes: Implicaciones clínicas. Arch Soc Esp Oftalmol [Internet]. Sociedad Española de Oftalmología; 2004. [citado 2015 Mar 24];79(8):38591. Disponible en: http:// scielo.isciii.es/scielo.php?script=sci_arttext\&pid=S0365-66912004000800006\&l- 
